\title{
The impact of a passenger-safety-driven acceleration limit on the operation of a bus service
}

\begin{abstract}
Buses are a form of active transportation and can improve people's well-being. However, their high level of acceleration can make them less attractive to users. Even worse, they can be responsible for severe injuries that require hospitalisation or for the development of fear of falling, particularly experienced by older people. Evidence has shown that, bus acceleration up to $1.0 \mathrm{~m} / \mathrm{s}^{2}$ enables passengers to move in a natural way inside the moving vehicle, hence reducing instability and increasing safety. Although operators might be willing to implement such an intervention, they might also be skeptical about its impact on the operation of a service, such as timetabling, travel times, waiting times etc.

The effect of a safety-driven acceleration limit on the operational characteristics of a round trip of a bus service in London is investigated by this study. Data regarding speed, acceleration and journey time were extracted from the engine of a bus and recorded at $2 \mathrm{~Hz}$. Further computations estimated the passenger waiting times and headways between the examined bus and its preceding and following buses. A vehicle movement model was used to test how these operational characteristics would be affected if the acceleration limit of $1.0 \mathrm{~m} / \mathrm{s}^{2}$ were to be implemented. The results suggest that the journey time of the proposed accessible service would be 6 min longer than the current service and passenger waiting time would increase by 2 min. One additional bus would be required to serve the same number of passengers. A discussion of the results is provided.
\end{abstract}

Keywords: bus acceleration; passenger safety; non-collision injuries; passenger waiting times; bus journey times; headway variation

\section{Introduction}

"An active city is a competitive city" is a motto that shapes the ideology of many city officials around the world and guides their strategic urban and transport planning for societal, economic and environmental growth (Designed to move, 2015). Being physically active has been scientifically associated with the improvement of people's health and well-being when compared to the excessive use of cars (Frank et al., 2004) and forms the basis of global campaigns focused on healthier future societies (WHO, 2018a).

Choosing active transport modes for the completion of everyday activities greatly contributes towards achieving the activity recommendation for a healthier lifestyle (WHO, 2018b). However, it is likely to be the case that some journeys for some people will require the use of public transport to bring them to the activities they are choosing, or have to, make. This is primarily because of distance (where the distance required to reach the activity is too great for the person to walk), or for other reasons (e.g. the need to carry shopping or other baggage, inclement weather, and so on). Buses are particularly important in these cases, mainly because of the density of their networks. Even where their network is sparse, it is likely to be nearer to people's origins and destinations than other public transport modes. Besides the fact that it is the most widespread public transport network in the world, the bus system is also the most cost-effective means of mobility for people of all age groups (Karekla and Tyler, 2019). 
Cost-effectiveness and a healthier lifestyle, however, do not seem to be factors that affect people's choices when it comes to commuting. Passenger cars are still the most preferred mode of travelling (83\%), whereas people use buses and coaches $(9.2 \%)$ more than trains $(7.6 \%)$ for their everyday movements (Eurostat, 2018). Nonetheless, bus journeys have been fluctuating since the beginning of the previous decade, with the lowest demand in Europe recorded in 2009 (Eurostat, 2018) and in the UK in 2014 (TfL, 2018).

Looking into the reasons why people still prefer their cars over the bus service and why bus passenger mileage is reducing, an official survey, that was carried out in London and interviewed 11,000 passengers, revealed that $25 \%$ of bus passengers are dissatisfied with the speed and acceleration of the bus. According to regular bus users, this is the third most important area that requires improvement and comes after the punctuality (31\%) and frequency (29\%) of the service (page 20, London Travel Watch (2010)). Due to abrupt bus movements, people are involved in non-collision accidents as they lose their balance, which in older passengers might result, not only in physical injuries, but also in fear of falling and avoidance of participating in societal activities.

Non-collision injuries aboard buses are at dramatic levels and affect passenger demand for bus services around the world. In Sweden, more than half of the recorded injuries on buses were caused by non-collision accidents (Björnstig et al., 2005) whereas in Portland Oregon, USA $80 \%$ of non-collision incidents involved loss of balance, with some of them occurring during the bus movement (Strathman et al., 2010). Moreover, the 3000 falls recorded every year during non-collision accidents on buses in the UK for those over 65 years old (Kendrick et al., 2015) reinforce the work of (Green et al., 2014) which states that the current bus service is dangerous and not designed to accommodate the needs of elderly users. Similar statistics can be found for other countries in Europe and in the world in the work carried out by (O'Neill, 2016).

Bus accelerations of levels higher than $2.0 \mathrm{~m} / \mathrm{s}^{2}$ are considered extremely dangerous for standing passengers whose balance is jeopardised in the case they do not get hold of a handrail (Browning, 1972; Dorn, 1998). Investigating the level of acceleration at which the London bus service operates, accelerations of up to $2.5 \mathrm{~m} / \mathrm{s}^{2}$ are recorded by the official operator (Sale, 2007) and are confirmed by the users (Karekla and Tyler, 2018b). Although extensive work is being done by transport operators worldwide to reduce the environmental impact of bus services, by introducing hybrid and full-electric buses that can control the way buses accelerate independently of the driver, still the acceleration levels are higher than the levels a healthy bus passenger could tolerate if they were to walk naturally inside a moving bus (Karekla and Tyler, 2018a).

Therefore, it is evident that a much lower level of bus acceleration of, for example, $1.0 \mathrm{~m} / \mathrm{s}^{2}$ should be sustained in order to increase accessibility and comfort during bus journeys, but also to increase patronage for this active mode of transportation (Karekla and Tyler, 2018b). More importantly, achieving a lower level of acceleration will reduce, and ideally eliminate, bus passenger injuries which would, as a consequence, reduce the substantial costs associated to it as a result of medical treatment and loss of earnings. In the UK, £2.3 billion were spent in 2015 to cover fall-related costs (NHS, 2017). The equivalent cost of falls in the USA in the same year reached US $\$ 50$ billion (CDC, 2019), which shows the importance and global applicability of this work.

Bus operators might expect that conforming to the recommended acceleration level will come at a cost, such as increased travel times and uneven headways that result in bus bunching (see Gkiotsalitis and Maslekar (2018a)). The work presented in this paper focuses on these aspects and investigates the impact of imposing a maximum, safety-driven acceleration level to a bus service in London. This investigation is performed using real-time CAN bus data from the drive system of a bus that indicates its acceleration, deceleration and trajectory. In addition, the 
expected trajectory of the same bus is generated, using an extension of the mathematical model of Fu et al. (2003) when imposing a maximum, safety-driven acceleration limit. The trade-off between improving safety and reducing the operational efficiency is investigated, e.g. increased bus journey time.

This paper is structured as follows: in section 2, relevant studies on the operational characteristics related to the bus service efficiency are being reviewed to investigate the trade-off between safety and operational costs. The contribution of this work to the scientific field is provided. Section 3 details the examined case study and the performance of current operations that do not impose an acceleration limit to bus trips. Section 4 investigates the effect of imposing lower accelerations on the operational efficiency of the bus service by using an extension of the mathematical model of Fu et al. (2003). Section 5 discusses the results and provides the limitations of this work, as well as recommendations for future work.

To avoid confusion, it is important to point out that wherever acceleration is being used here forth is done for simplification and both acceleration and deceleration phases are implied.

\section{Background}

\subsection{Operational characteristics related to bus service efficiency}

Imposing limits on acceleration levels might increase the travel times of bus trips. This will have an effect on the passenger travel times, the trip dispatches (which might be delayed resulting in "schedule sliding"), the operational headways and the vehicle and crew schedules (Li et al., 2009; Cats et al., 2011; Gkiotsalitis and Cats, 2018). The adverse effects of increased travel times have been acknowledged scientifically and, as a solution, it has been proposed to proactively embed slack times to the bus schedules to cater for unexpected delays (Yan et al., 2006; Xuan et al., 2011; Daganzo, 2009; Adamski and Turnau, 1998; Zhao et al., 2006).

Apart from adding slack times, real-time control measures such as stop-skipping (Chen et al., 2015; Yu et al., 2015; Sun and Hickman, 2005) and short-turning (Zhang et al., 2017; Gkiotsalitis et al., 2019b) can be deployed to reduce the travel times of specific bus trips. Nevertheless, short-turnings can increase the deadheading times of buses and cause passenger inconvenience because of the need to wait for another one. Stop-skippings generate a sense of unreliability and inconvenience for waiting passengers who are unable to board buses (Liu et al., 2013), and in any case can only really be implemented if there are no passengers on the bus wishing to alight at the skipped stops. Therefore, stop-skipping is a very unreliable tactic.

Increased travel times, because of acceleration limits, can also impact the synchronisation of bus services with other bus services or trains. This is reported in a distinct line of works, which have been focused on bus schedule synchronisation (including Ceder et al. (2001); Cevallos and Zhao (2006); Ibarra-Rojas and Rios-Solis (2012); Wei and Sun (2017); Gkiotsalitis and Maslekar (2018b); Gkiotsalitis et al. (2019a)). In particular, increasing the inter-station travel times of buses might result in delayed arrivals at transfer stops and missed passenger connections. In addition to that, increased trip travel times because of the safety-driven acceleration limits might require the deployment of more vehicles to maintain the same frequency level. This can result in an increased fleet size that should be addressed at the tactical planning stage (Yu et al., 2010; Verbas et al., 2015; Gkiotsalitis and Cats, 2018; Sun and Szeto, 2019).

Apart from the impact to trip travel times, lower accelerations might also degrade the regularity of bus services. Especially in high-frequency services, such as bus services with frequencies of more than 5 buses per hour, the main objective is to reduce the variation between actual and scheduled waiting times of passengers for increased service regularity (Trompet et al., 2011). The actual arrival times of buses at stops are monitored with the use of telematics; this enables transport authorities to penalise under-performing bus operators and reward those that perform 
the best (Jansson and Pyddoke, 2010). Incentivising bus operators to improve service regularity helped to reduce the expected waiting times for passengers in London. In particular, the expected passenger waiting times are used as a key performance indicator for measuring service regularity in high-frequency services. They are modelled considering half the average headway among successive buses at the stops of the line plus the variance of those headways. This is then divided by two times the average headway resulting in the expected passenger waiting times (see Newell and Potts (1964); Trompet et al. (2011)). In London, excess waiting times have been reduced from 4 minutes in 1979 to 1.2 minutes in 2012 to 1.1 minutes in 2017 (TfL, 2017). Interestingly, this has been done through centralised control of headways, by requiring drivers to wait at bus stops if they are running too close to the preceding bus. This put pressure on the driver, which could result in them offsetting their frustration possibly through high rates of acceleration.

It is evident that there is an increased pressure on bus drivers to adjust their speeds and accelerate beyond the safety-recommended levels in order to meet the operational key performance indicators (this is also noted in Koehler et al. (2011); Daganzo and Pilachowski (2011)). Notwithstanding, to the best of the authors' knowledge, past works on improving bus operations (i.e., travel times and waiting times of passengers) do not consider the adverse effects to the passenger safety due to abnormal accelerations (Eberlein et al., 2001; Chen et al., 2013; Gkiotsalitis, 2020).

\subsection{Contribution of this study}

This study is investigating the impact of a safety-driven acceleration on two main key performance indicators for bus services:

1. the regularity of the service, which indicates the time passengers would need to wait at a bus stop before a bus arrives; and

2. bus journey time, which indicates the travel time of a bus along the route, as well as the time passengers will spend aboard that bus before reaching their destination.

This is the first time the impact of a maximum bus acceleration level is investigated. The study aims to contribute to the public transport field by promoting active commuting. Improving bus services by reducing non-collision passenger accidents, would make buses more attractive to potential users, with the added benefit that service operators will not have to suffer great additional expenses to achieve this. The outcomes of this work would benefit bus users and operators around the world, especially of bus services operated in densely populated areas. The impact of an acceleration limit on the above performance indicators, would generally be greater on high-frequency bus routes.

Thus, this study focuses on a high-frequency bus route in a densely populated area in London. Using CAN bus data from a single bus we report the service regularity, travel time, and acceleration/deceleration of the bus every 2 seconds. The well-established model of Fu et al. (2003) is then employed to investigate how a safety-driven acceleration limit can impact the service regularity and journey time.

\section{Performance of the Current Bus Service: the London Case Study}

Imposing an acceleration limit to a bus service, that would increase passenger safety aboard buses, can have an impact on the operations in cities with intense bus services, such as London, Ottawa, Hong Kong, or Singapore. To investigate this effect, bus route 388 of the London bus system was examined. This route is operated by HCT Group, one of London's biggest bus operators. It is a daily bi-directional bus route, operated at a high frequency (every 10-12 min) 
that starts in Stratford City and ends in Elephant \& Castle. It serves 36 bus stops in some of the densest parts of London (i.e. Stratford, Shoreditch, Liverpool Street). The topology of the bus line is provided in Figure 1 where the line layout and the 15 most important bus stops are presented. The total length of the route over both directions is $22.56 \mathrm{~km}$.

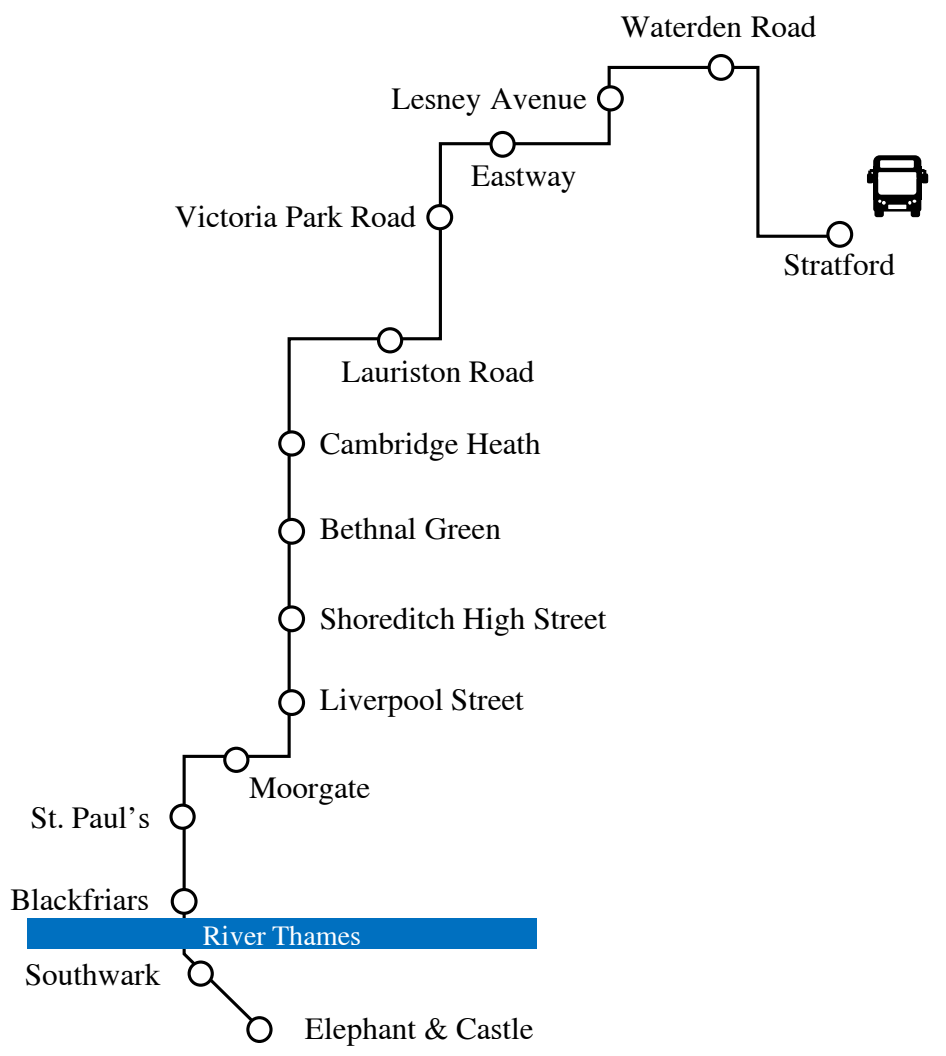

Figure 1: Main stops of bus route 388 between Stratford City and Elephant \& Castle

The first buses arrive at the Stratford City bus stop as early as 05:25 and the last ones at 23:50 on both weekdays and weekends. In the other direction, the service from Elephant \& Castle starts at 05:45 with the last trip occurring at 00:40. Successive buses in both directions are dispatched with a headway that varies between 9 and 14 minutes depending on the peak and off-peak time of the day.

To investigate the smoothness of the current service, real-time accelerations and decelerations from the drive system of one bus serving this route were collected. The bus is owned by the UCL PAMELA Laboratory, it is an Alexander Dennis Enviro 400H hybrid bus and serves the 388 bus service of Transport for London (TfL), when it is not needed by the University for experimental work. Complete data were obtained from one round-trip performed by the UCL hybrid bus on a Saturday from 11:21 until 13:38, whilst the bus was running a normal service.

Specifically, vehicle speed data were collected every 2 seconds, from which acceleration and deceleration was calculated. The total duration of the data collection is $2 \mathrm{~h}$ and $17 \mathrm{~min}$ and includes an entire round-trip. The high-granularity data were exported from the vehicle drive system, digitised and organised into a database for further manipulation. To identify which data were associated with the bus being in motion and which with the bus being idle at bus stops or traffic lights, the bus acceleration and deceleration were analysed.

Based on the acceleration data, the bus was in motion for $5754 \mathrm{~s}$ and idle for $2644 \mathrm{~s}$ (re- 
sulting in $8398 \mathrm{~s}$ of total time of data collection). The bus was completely stopped 109 times (acceleration $=0 \mathrm{~m} / \mathrm{s}^{2}$ ) in 36 traffic lights and 72 bus stops. At one instance, around 13:13:34, the bus was stationary for $153 \mathrm{~s}$ because of a change of driver shifts. The final database, part of which is presented in Table 1, includes variables such as time, the bus speed and acceleration, vehicle status (running or stopped), and stop duration.

Table 1: Example of CAN bus data collected every 2 seconds

\begin{tabular}{cccccc}
\hline \multicolumn{1}{c}{ Timestamp } & & & Speed & Acc/Dec & Status \\
\cline { 1 - 2 } \cline { 5 - 6 } hh:mm:ss & sec & & $\mathrm{m} / \mathrm{s}$ & $\mathrm{m} / \mathrm{s}^{2}$ & \\
\hline $11: 22: 16$ & 40936 & & 0.77 & -1.309 & running \\
$11: 22: 18$ & 40938 & & 0 & -0.386 & running \\
$11: 22: 20$ & 40940 & & 0 & 0 & idle (bus stop) \\
$11: 22: 22$ & 40942 & & 0 & 0 & idle (bus stop) \\
\hline
\end{tabular}

The observed dwell time at each bus stop varied between 4 and 22 seconds. TfL provides three sets of open access information of the bus arrival times (Monday to Friday, Saturday and Sunday and Bank holidays timetables) at 12 key bus stops along the route, known as control point stops. The appropriate timetable was matched with the data observed from the bus driving system at these control point stops, and the regularity of the service was evaluated with the use of a specific key performance indicator; the expected passenger waiting time. In addition, the total round-trip travel time, which indicates the travel times of passengers aboard the bus, is also provided.

As mentioned earlier, in this work, the enforcement of a safety-driven acceleration limit and its impact on these two key performance indicators are studied. It should be noted at this point that the crowding level in the bus can be an additional key performance indicator, however such data were not collected.

The acceleration of the examined bus was monitored every 2 seconds and the observed values are presented in Fig.2.

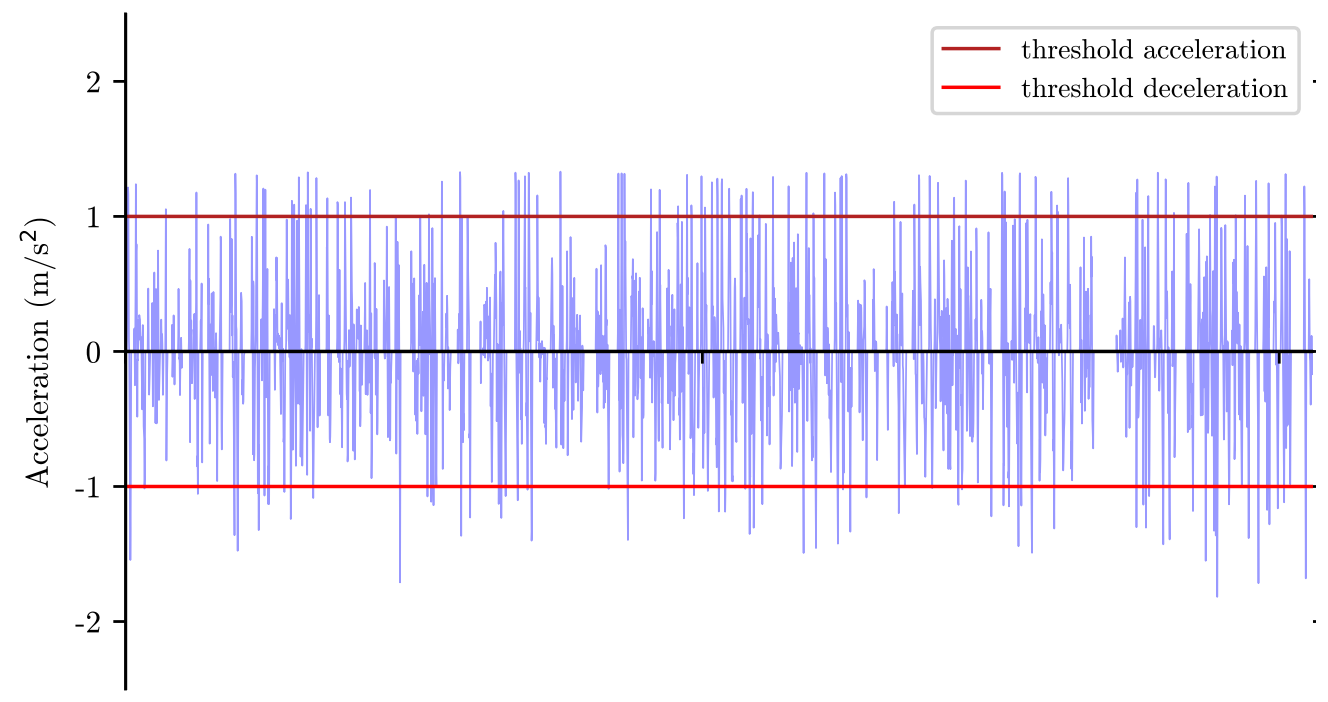

Figure 2: Observed bus acceleration on the examined round trip

In Fig.2 there are several instances where the acceleration or the deceleration are more than $1.0 \mathrm{~m} / \mathrm{s}^{2}$, which is the acceleration limit recommended by Karekla and Tyler (2018b). This indicates that the current service is not smooth and affects the safety and comfort of passengers. 
To further investigate the occurrence of abrupt acceleration and deceleration, the frequency of their exceedance above $1.0 \mathrm{~m} / \mathrm{s}^{2}$ is reported in Table 2. It is worth noting that, whilst the greatest acceleration magnitude is in the deceleration phase (minimum observed deceleration is $1.82 \mathrm{~m} / \mathrm{s}^{2}$ ), the majority of instances when acceleration exceeded the $1.0 \mathrm{~m} / \mathrm{s}^{2}$ threshold occurs in the acceleration phase (132 occurrences). Although deceleration might not be in the control of the driver so much, as they might be avoiding a collision, acceleration is very much under their control and highlights the importance of this study.

Table 2: Statistics of collected bus data

\begin{tabular}{lllll}
\hline $\begin{array}{l}\text { Total } \\
\text { Observations }\end{array}$ & $\begin{array}{l}\text { Maximum observed } \\
\text { Acceleration }\end{array}$ & $\begin{array}{l}\text { Maximum observed } \\
\text { Deceleration }\end{array}$ & $\begin{array}{l}\text { Occurrences } \\
\left(\operatorname{acc}>1.0 \mathrm{~m} / \mathrm{s}^{2}\right)\end{array}$ & $\begin{array}{l}\text { Occurrences } \\
\left(\mathrm{dec}<-1.0 \mathrm{~m} / \mathrm{s}^{2}\right)\end{array}$ \\
\hline 4117 & $1.33 \mathrm{~m} / \mathrm{s}^{2}$ & $-1.82 \mathrm{~m} / \mathrm{s}^{2}$ & $132(3.21 \%)$ & $97(2.35 \%)$ \\
\hline
\end{tabular}

The total travel time of the examined round-trip is 137 minutes and 12 seconds. A final key performance indicator is the service regularity which, in high-frequency services, is calculated in the form of expected (i.e., average) passenger waiting times (Trompet et al., 2011). The measure of instability of the expected passenger waiting times is the coefficient of variation of the actual headways. Assuming random passenger arrivals at stops follow the Poisson distribution, the expected passenger waiting times are directly proportional to the coefficient of variation of headways, $H$, and are expressed by the relation of Newell and Potts (1964):

$$
\mathbb{E}[W] \doteq \frac{\mathbb{E}[H]}{2}+\frac{\operatorname{Var}[H]}{2 \mathbb{E}[H]}
$$

where $\mathbb{E}[W]$ is the average expected waiting time and $\operatorname{Var}[H]$ the headway variance. To compute the average expected waiting time of passengers, the time headways between the examined trip and its preceding and following trip were plotted for every control point stop. Those plots are presented in Fig.3 where the left sub-plot refers to the direction from Elephant \& Castle to Stratford City and the right sub-plot to the direction from Stratford City to Elephant \& Castle. 

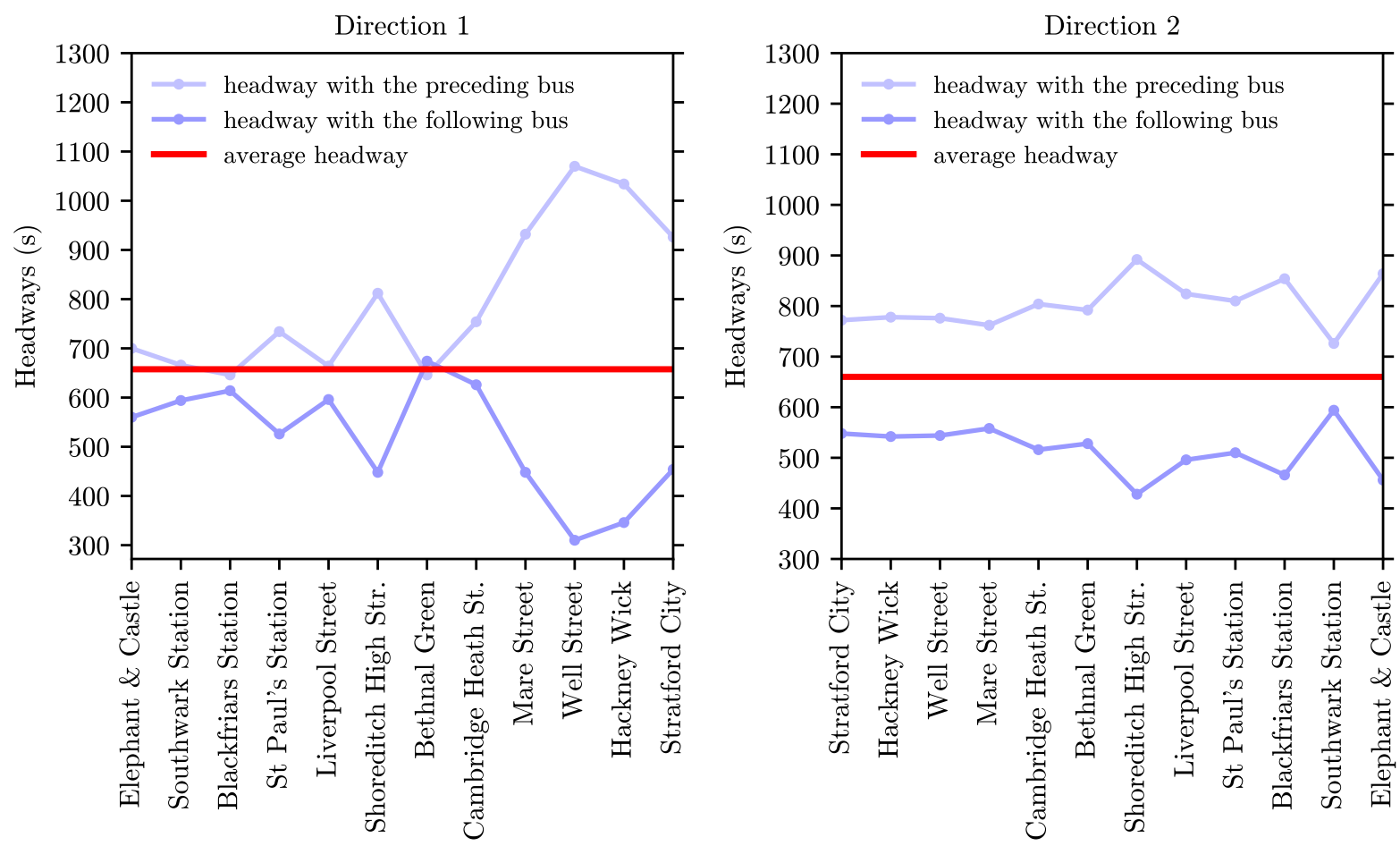

Figure 3: Observed time headways between the examined bus and its preceding and following buses at each control point stop, compared with the average headway of the examined bus

From Fig.3 it is evident that the examined bus was left behind after the Cambridge Heath Station control point stop when it was operating in direction 1. Indeed, after Cambridge Heath Station its time headway with its preceding bus was in the range of 900-1100s and with its following in the range of 200-500s. This clearly indicates that the examined bus and its following bus were bunching together. In direction 2 (shown in the right sub-figure), although the time headway between the examined bus and its preceding and following buses is not near the average headway, no significant bunching problem was observed. The time headway between the examined and its following bus was persistently shorter than the time headway between the examined and its preceding bus, which was consistently above 2 minutes. Using the average observed headway between the examined bus and its preceding and following buses, passenger waiting time was calculated at every stop, as presented in Fig.4. As expected, the expected waiting time of passengers in direction 1 is significantly higher than the respective one in direction 2. The problematic control point stops are the ones after Cambridge Heath Station where passengers have to wait for more than $6.4 \mathrm{~min}$ on average, and up to $7.5 \mathrm{~min}$ in the worst case (Well Street bus stop). 

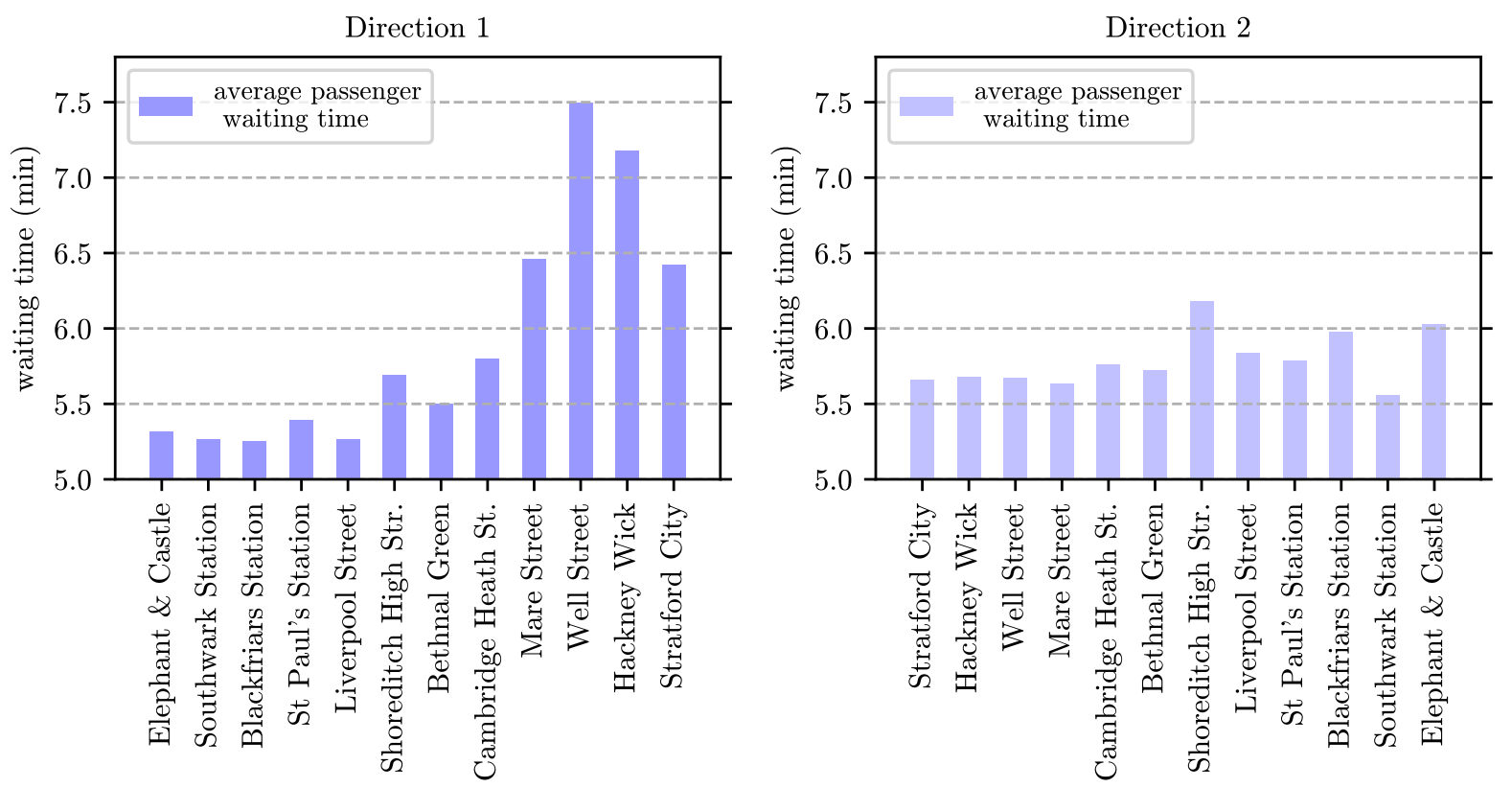

Figure 4: Average expected passenger waiting time between the experimental bus trip and its preceding/following trip at each control point stop

\section{Operational impact of the safety-driven acceleration limit}

To investigate the impact of the safety-driven acceleration limit of $1.0 \mathrm{~m} / \mathrm{s}^{2}$ on the examined bus trip, a simulation using the well-established model of Fu et al. (2003), that generates vehicle trajectories with the use of acceleration / deceleration data, was performed. The model of Fu et al. (2003) is employed as it serves the purpose of this work: it allows the simulation of bus trajectories based on different acceleration profiles and can estimate new bus trajectories when an acceleration limit is imposed. The simulated bus trajectories when the safety-driven acceleration limit is imposed are then used to estimate the impact on bus travel time, passenger waiting time, and the required fleet size.

The vehicle movement model of Fu et al. (2003) relies on the following assumptions:

1. Buses that serve the same line do not overtake each other. This is an assumption used in several vehicle movement models (refer to Xuan et al. (2011); Chen et al. (2013)) and as time headways between the examined bus and its preceding and following buses in the examined case study are never zero (see Fig.3) we can accept this assumption as reasonable;

2. Passenger arrival time at bus stops is considered random. For bus services with high frequency $(\leq 15 \mathrm{~min}$ ), like the one described in this work, passengers cannot coordinate their arrival at a bus stop with the arrival time of a bus at the same bus stop(Welding (1957); Randall et al. (2007));

3. When imposing an acceleration limit, changes to the bus service travel time depend only on the delay resulting from the upper-bound of that acceleration limit.

The new trajectory of the examined bus, after imposing the safety-driven acceleration limit, is generated with the extension of the vehicle movement model (nomenclature is introduced in Table 3), which is discussed below. 
Table 3: Nomenclature of vehicle movement model parameters

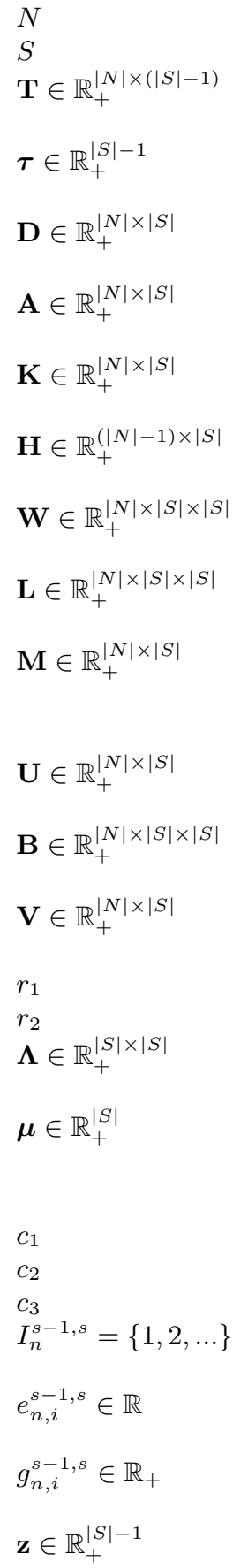

set of bus trips, $N=\{n-1, n, n+1\}$, where $n$ is the examined bus;

set of bus stops, $S=\{1, \ldots, s, \ldots,|S|\}$;

matrix of running times where $t_{n, s} \in \mathbf{T}$ is the running time of the $n$-th trip between stop $s-1$ and $s$ where $s \in S \backslash\{1\}$;

vector of free-flow running times $\boldsymbol{\tau}=\left(\tau_{2}, \ldots, \tau_{|S|}\right)$ where $\tau_{s}$ is the free-flow running time between stop $s-1$ and $s$ where $s \in S \backslash\{1\}$;

matrix of departure times where $d_{n, s}$ is the departure time of trip $n$ from stop $s$ where $n \in N$ and $s \in S$;

matrix of arrival times where $a_{n, s}$ is the arrival time of trip $n$ at stop $s$ where $n \in N$ and $s \in S$;

matrix of dwell times where $k_{n, s}$ is the dwell time of trip $n$ at stop $s$ where $n \in N$ and $s \in S$;

matrix of bus headways times where $h_{n, s}$ is the headway between trips $n-1$ and $n$ at stop $s$ where $n \in N \backslash\{1\}$ and $s \in S$;

matrix where each $w_{n, s y} \in \mathbf{W}$ denotes the number of passengers waiting for bus $n$ and traveling from stop $s$ to $y$ (note: $w_{n, s y}=0, \forall y \leq s$ );

matrix where each $l_{n, s y} \in \mathbf{L}$ denotes the number of passengers traveling from stop $s$ to stop $y$ skipped by bus $n$ (note: $l_{n, s y}=0, \forall y \leq s$ );

matrix where each $m_{n, s} \in \mathbf{M}$ denotes the number of passengers at stop $s$ skipped by bus $n$ where $n \in N, s \in S$ (note: $m_{n, s}=\sum_{i=s+1}^{|S|} l_{n, s i}$ );

matrix where each $u_{n, s} \in \mathbf{U}$ denotes the number of passengers boarding bus $n$ at stop $s$ where $n \in N, s \in S$ (note: $u_{n,|S|}=0, \forall n \in N$ );

matrix where each $b_{n, s y} \in \mathbf{B}$ denotes the number of passengers boarding bus $n$ at stop $s$ whose destination is stop $y$ (note: $b_{n, s y}=0, \forall y \leq s$ );

matrix where each $v_{n, s} \in \mathbf{V}$ denotes the number of passengers alighting bus $n$ at stop $s$ where $n \in N, s \in S$ (note: $v_{n, 1}=0, \forall n \in N$ );

average boarding time per passenger, a constant;

average alighting time per passenger, a constant;

matrix where each $\lambda_{s y} \in \boldsymbol{\Lambda}$ denotes the average passenger arrival rate at stop $s$ whose destination is stop $y$ (note: $\lambda_{s y}=0, \forall 1 \leq y \leq s \leq N$ );

vector where each $\mu_{s} \in \boldsymbol{\mu}$ denotes the average passenger arrival rate at stop $s$ (note:

$\left.\mu_{s}=\sum_{i=s+1}^{|S|} \lambda_{s i}\right)$

unit time value associated with the passenger waiting times $(\$ / \mathrm{h})$;

unit time value associated with the passenger in-vehicle travel time $(\$ / \mathrm{h})$;

unit time value associated with vehicle operation time $(\$ / \mathrm{h})$;

is a set denoting the frequency of occurrence of a measurement/observation of the instantaneous acceleration of trip $n$ when a bus travels from stop $s-1$ to stop $s$;

the instantaneous acceleration of trip $n \in N$ according to the $i$-th measurement, where $i \in I_{n}^{s-1, s}$ and the bus trip $n$ travels from stop $s-1$ to stop $s$;

the instantaneous speed of trip $n \in N$, where $i \in I_{n}^{s-1, s}$ and the bus trip $n$ travels from stop $s-1$ to stop $s$;

vector where each $z_{s} \in \mathbf{z}$ denotes the travel distance between bus stop $s-1$ and $s$ in meters.

\subsection{Vehicle movement model}

The new trajectory of the examined bus, after imposing the safety-driven acceleration limit, is generated with the extension of the vehicle movement model of Fu et al. (2003). In the vehicle movement model, the arrival time of the examined bus trip $n$ at stop $s$ is equal to its departure time at stop $s-1\left(d_{n, s-1}\right)$ plus the travel time between the two stops:

$$
a_{n, s}=d_{n, s-1}+t_{n, s}, \forall s \in S \backslash\{1\}
$$


In addition, the departure time of the examined trip $n$ from stop $s$ is equal to its arrival time plus the dwell time $k_{n, s}$ :

$$
d_{n, s}=a_{n, s}+k_{n, s}, \forall s \in S \backslash\{1\}
$$

Assuming that overtaking between buses of the same line is not allowed, the departure headway between bus trip $n$ and its preceding one reads:

$$
h_{n, s}=d_{n, s}-d_{n-1, s}, \forall n \in N \backslash\{n-1\}, s \in S
$$

The dwell time of each bus trip $n$ at each stop $s$ depends on the number of passengers who will board and alight at the stop, denoted by $u_{n, s}$ and $v_{n, s}$, respectively:

$$
k_{n, s}=r_{1} u_{n, s}+r_{2} v_{n, s}, \forall n \in N \backslash\{n-1\}, s \in S \backslash\{1\}
$$

The expected number of passengers who will board bus trip $n$ at stop $s$ (assuming bus $n$ stops at stop $s$ ) depends on the number of passengers traveling between stops $s$ and $y(y>s)$ :

$$
u_{n, s}=\sum_{y=s+1}^{|S|} w_{n, s y}, \forall n \in N \backslash\{n-1\}, s \in S \backslash\{|S|\}
$$

where $w_{n, s y}$ is the number of passengers waiting for bus $n$ and traveling from stop $s$ to $y$.

The expected number of alighting passengers for bus trip $n$ at stop $s$ depends on the number of passengers traveling between stops $y$ and $s(y<s)$ :

$$
v_{n, s}=\sum_{y=1}^{s-1} w_{n, s y}, \forall n \in N \backslash\{n-1\}, s \in S \backslash\{1\}
$$

It is important to highlight that Eqs.(2)-(7) are based on the model of Fu and Yang (2002), which is further expanded in this work to consider the effect of instantaneous acceleration/deceleration on the inter-station running times. In particular, let $e_{n, i}^{s-1, s}$ be the $i$-th observation of the instantaneous acceleration of the examined bus $n$ that travels from stop $s-1$ to stop $s$ (thus, $\left.i \in I_{n}^{s-1, s}\right)$. A new measurement of the instantaneous acceleration was collected every 2 sec for the examined bus. Therefore, each observed instantaneous acceleration $e_{n, i}^{s-1, s}$ where $i \in I_{n}^{s-1, s}$ refers to the (very short) time period $[i, i+2 \mathrm{sec}$ ). Assuming that the observed instantaneous acceleration $e_{n, i}^{s-1, s}$ does not deviate significantly within each time period $[i, i+2 \mathrm{sec})$, the instantaneous speed at each instance $i \in I_{n}^{s-1, s}$ can be derived as:

$$
g_{n, i}^{s-1, s}=\left\{\begin{array}{l}
g_{n, 1}^{s-1, s} \text { if } i=1 \\
g_{n, i-1}^{s-1, s}+\int_{i}^{i+2} e_{n, i}^{s-1, s} \mathrm{~d} t, \forall i \in I_{n}^{s-1, s} \backslash\{1\}
\end{array}\right.
$$

where $\int_{i}^{i+2} e_{n, i}^{s-1, s} \mathrm{~d} t=2 e_{n, i}^{s-1, s}(\mathrm{~m} / \mathrm{s})$. Eq.8 denotes that the instantaneous speed $g_{n, i}^{s-1, s}$ of our trip $n$ when it departs from any stop $s-1 \in S \backslash\{1\}$ is initially $g_{n, 1}^{s-1, s}$, where $g_{n, 1}^{s-1, s}=0(\mathrm{~m} / \mathrm{s})$ 
if bus trip $n$ stopped at bus stop $s$, and is updated by adding the integral of the observed instantaneous acceleration to the previously calculated value of the instantaneous speed, $g_{n, i-1}^{s-1, s}$.

Based on the above, the running time of the examined bus $n \in N$ from any bus stop $s-1$ to bus stop $s$ where $s \in S \backslash\{1\}$ can be calculated as:

$$
t_{n, s}=z_{s}\left[\frac{g_{n, 1}^{s-1, s}+\sum_{i=2}^{\left|I_{n}^{s-1, s}\right|}\left(g_{n, i-1}^{s-1, s}+\int_{i}^{i+2 s e c} e_{n, i}^{s-1, s} \mathrm{~d} t\right)}{\left|I_{n}^{s-1, s}\right|}\right]^{-1}
$$

where

$$
\frac{g_{n, 1}^{s-1, s}+\sum_{i=2}^{\left|I_{n}^{s-1, s}\right|}\left(g_{n, i-1}^{s-1, s}+\int_{i}^{i+2 s e c} e_{n, i}^{s-1, s} \mathrm{~d} t\right)}{\left|I_{n}^{s-1, s}\right|}
$$

is the average speed of trip $n$ between stops $s-1$ and $s$ according to the actual measurements of the instantaneous acceleration.

\subsection{Results}

Our experiments are performed in a general-purpose computer with Intel Core i7-455 7700HQ CPU @ 2.80GHz and 16 GB RAM. Replacing the instantaneous accelerations in Eq.9 with the acceleration limit of $1.0 \mathrm{~m} / \mathrm{s}^{2}$ for those accelerations that exceed the safety-driven limit, results in an updated trajectory for the examined bus.

Fig.5 shows the actual trajectory of the examined bus before imposing the safety-driven acceleration limit and the expected trajectory in the case where this limit is imposed, when performing the round-trip from Elephant \& Castle Station to Stratford City and back. This exercise assumes that the traffic conditions pertaining to the presence of the bus, and thus affecting (i) its movement, (ii) the time taken for passenger boarding and alighting, and (ii) the time taken to change drivers, are all the same for both cases. That is, the only change between these two cases is the acceleration rates.

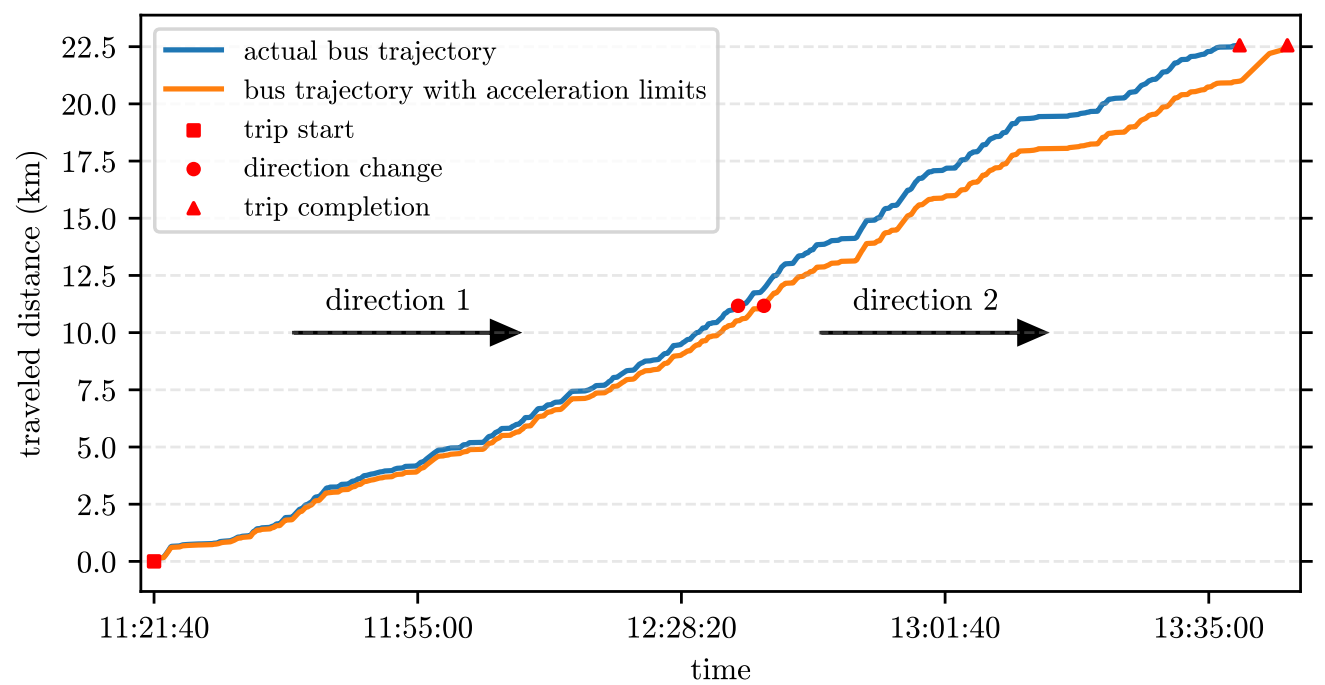

Figure 5: Actual and estimated trajectory of the examined bus with and without the proposed acceleration limit

From Fig.5 one can note that in the actual operations the examined bus started its journey at 11:21:40, arrived at Stratford City around 12:34:00, and completed its round-trip at 13:38:52. 
Additionally, as previously discussed, around 13:13:34 the bus remained idle for $153 \mathrm{~s}$ because of a change of driver shifts. When the acceleration limit is imposed, the examined bus is expected to take 6 min longer to complete the same round trip, arriving at the final stop at 13:44:54.

Besides the extension of the total travel time in each direction, which affects passenger travel times and delays the dispatch of the bus on its next journey, imposing the safety-driven acceleration limit could have an impact on the regularity of bus services. This study considers the worst-case scenario where the examined trip is constrained to use the safety-driven acceleration limit whilst its preceding and following buses operate as usual. This assumes that there is no alteration in the number of passenger boardings and alightings resulting from this change on any of the three discussed buses. The extreme situation studied in this paper is not expected in practice as in the case a bus operator implements an acceleration limit, this will apply to all buses serving the route. Nevertheless, this work measures the worst-possible impact to the service regularity when only one trip complies with the recommended acceleration limit. The results are presented in Fig. 6 and are expressed in terms of average expected passenger waiting times at every control point stop. As direction 1 presented worse results than direction 2 when the current service was analysed, expected waiting times for the accessible service are focused on the worst performing of the two directions (direction 1).
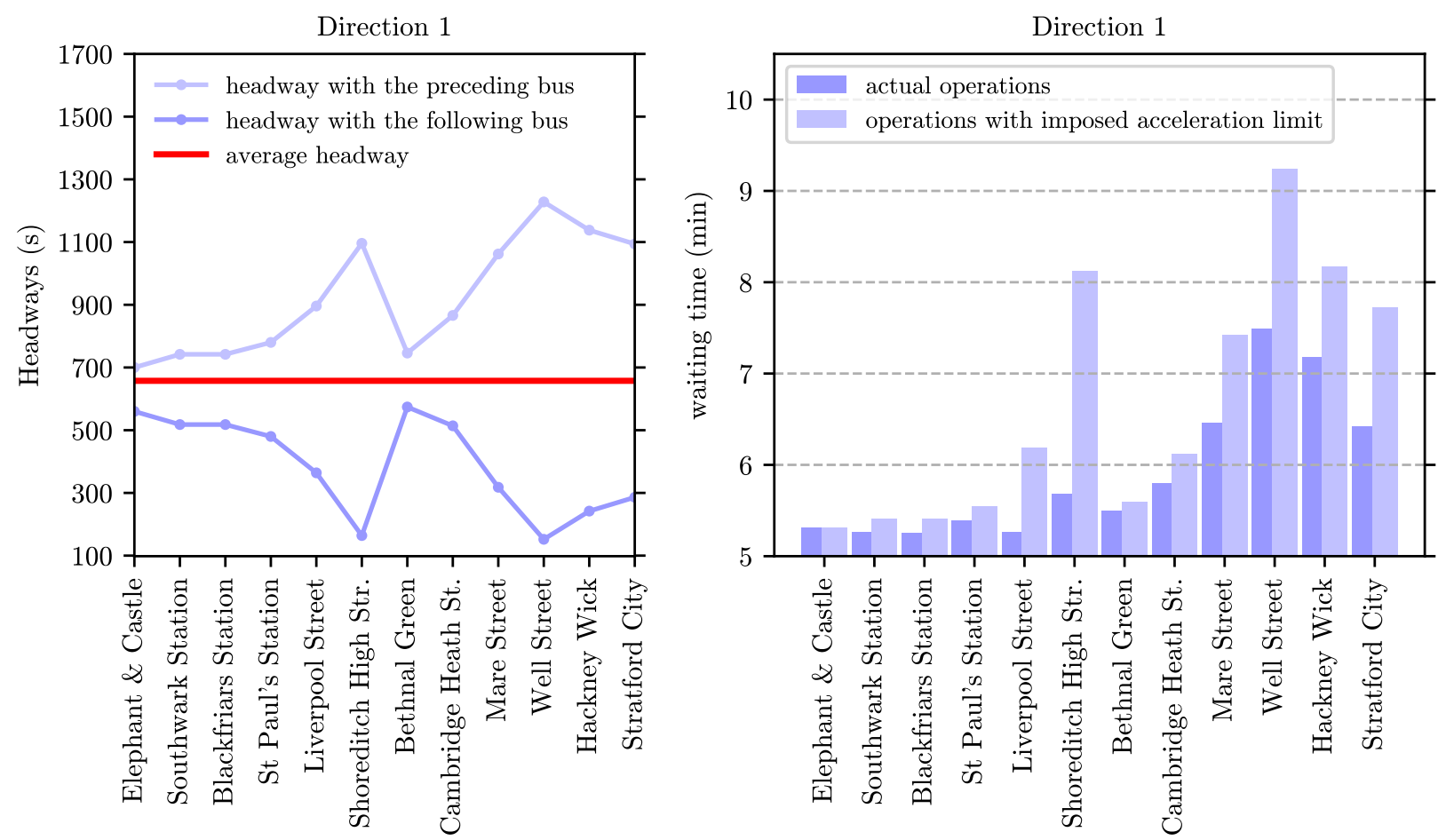

Figure 6: Time headways for the accessible service (left) and average expected passenger waiting time at each control point stop for the current and accessible services (right). Note that for the accessible service the acceleration limit of $1.0 \mathrm{~m} / \mathrm{s}^{2}$ was imposed to the examined bus only, not to its preceding and following buses.

The results of this experiment, are summarised in Table 4 and focus on the following four factors:

1. Travel time of the examined bus in the accessible service (when the proposed acceleration limit is implemented) is 6 min longer than bus travel time in the current situation. More specifically, passengers of the examined bus will be subjected to an approximately $3 \mathrm{~min}$ and $10 \mathrm{sec}$ longer journey in each direction;

2. Passenger maximum expected waiting time in the accessible service increases by 1.7 min 
compared to the current service;

3. The coefficient of variation $\left(\mathrm{CV}=\frac{\text { standard deviation }}{\text { mean }}\right)$ in directions 1 and 2 reveals that the instability of the expected passenger waiting times increases when the acceleration limit is imposed. In a perfectly regular service, the coefficient of variation would be equal to zero;

4. The number of violations of the recommended acceleration limit that might lead to collision and non-collision passenger injuries reveal that in the accessible service bus passenger safety increases; and

5. The required bus fleet to serve the examined bus route. Fleet size is calculated by $F_{S}=$ $\left\lceil T_{c} / H_{s}\right\rceil$, where $T_{c}$ is the total bus travel time to complete a round trip, and $H_{s}$ is the scheduled headway among trips (in this study, $H_{s}=11 \mathrm{~min}$ ). This shows that in the case an acceleration limit of $1.0 \mathrm{~m} / \mathrm{s}^{2}$ were to be implemented, one additional bus would be required to serve the same number of passengers.

Table 4: Performance summary of the current and accessible bus service

\begin{tabular}{|c|c|c|}
\hline & $\begin{array}{r}\text { Current Bus Service } \\
\left(\text { abs Max Acc }>1.0 \mathrm{~m} / \mathrm{s}^{2}\right)\end{array}$ & $\begin{array}{r}\text { Accessible Bus Service } \\
\left(\text { abs Max Acc }=1.0 \mathrm{~m} / \mathrm{s}^{2}\right)\end{array}$ \\
\hline$T_{c}:$ Total bus travel time & $137 \min 12 \mathrm{~s}$ & $143 \min 14 \mathrm{~s}$ \\
\hline Travel time in direction 1 & $72 \min 20 \mathrm{~s}$ & $75 \min 10 \mathrm{~s}$ \\
\hline Travel time in direction 2 & $64 \min 52 \mathrm{~s}$ & $68 \min 04 \mathrm{~s}$ \\
\hline $\mathrm{CV}$ of passenger waiting time - direction 1 & 0.29 & 0.47 \\
\hline $\mathrm{CV}$ of passenger waiting time - direction 2 & 0.23 & 0.39 \\
\hline Violations of recommended acc/dec limit & 229 & 0 \\
\hline Maximum expected waiting time & $7.5 \mathrm{~min}$ & $9.2 \mathrm{~min}$ \\
\hline$F_{S}:$ Required Fleet size & 13 & 14 \\
\hline
\end{tabular}

\section{Discussion}

Ensuring that bus services provide an increased level of accessibility and enable people's mobility to reach and pursue everyday activities is crucial for the health and well-being of future generations.

Bus operational characteristics have been in the spotlight for decades with researchers investigating ways to increase passenger satisfaction, a key element of attracting people in using buses for their everyday commutes (Shang et al., 2019), and to reduce bus emissions for a more positive environmental impact.

Although abrupt bus accelerations have been reported as one of the most disappointing elements associated with discouraging potential users from using the provided services, scientific work on this topic has followed a different path to increase bus passenger satisfaction.

Following Karekla (2016), it is clear that lower bus accelerations could be a way to attract people to use buses as part of their active travel. The impact of bus acceleration on the operational characteristics of the service, however, have only been considered as part of eco-driving Zeng et al. (2020), and strategies such as reducing bus headways (Berrebi et al., 2015), providing real-time information for the bus arrival times (Lu et al., 2018), redesigning a bus system to relocate bus stops or reduce the number of stops along a route (Shatnawi et al., 2020) and including dedicated bus lanes to achieve a consistent bus speed (He et al., 2019) have been investigated to increase passenger satisfaction. 
This paper identified this gap and considered the effects of reducing bus acceleration on the service regularity (passenger waiting time) and bus journey time, with the aim to change operator's current perception that such an accessible service would be unreliable.

The 388 bus service in London, operating along a 23km corridor, provided the platform to investigate the effect of a safety-driven acceleration limit of $1.0 \mathrm{~m} / \mathrm{s}^{2}$ on the operational characteristics of the service. Long bus journey times involve the risk of turning people away from using buses and as a result operators would avoid adopting lower acceleration levels in order to maintain their service demand. A reduced service demand would lead to less passengers, increased fares for the remaining patronage and therefore lead to an increase in car ownership and decline of people's physical and mental health. However, low passenger safety and high risk of non-collision accidents during bus journeys would have a similar effect.

Bus speed, acceleration and deceleration, as well as travel time for a round trip of the 388 service were recorded at $2 \mathrm{~Hz}$ and extracted from the bus drive system. The data were organised in a database, which revealed that around $5 \%$ of the acceleration data exceeded the safety-driven acceleration limit in both the acceleration and deceleration phases. Although these instances do not occur frequently, and are not sustained for prolonged periods throughout the bus journey, they are capable of causing severe imbalances and non-collision injuries to passengers aboard the bus. The fact that they also occur unexpectedly, adds to the problem and further reduces bus passenger safety. Hence, it is essential for bus services to operate at lower acceleration levels in order to provide a more accessible bus service.

With regards to the time headways between the examined bus and its preceding and following buses, it was shown that the service as operated on this occasion deviated from the published timetable and as a result the examined bus was operating at long headways from its preceding bus and at short headways from its following bus. It was also clear that the service operated differently in these respects at different places along the route. This was especially apparent in direction 1. It did not come as a surprise that passengers of the examined bus were waiting at bus stops for unusually long times that reached up to $7.5 \mathrm{~min}$ on average (Fig.3).

Applying the safety-driven acceleration limit of $1.0 \mathrm{~m} / \mathrm{s}^{2}$ only to accelerations and decelerations that exceeded this threshold, it was concluded that a bus of this service would require 6 min longer to complete the round trip. At the same time waiting times at some stations could reach 9 min on average (Fig.6) which would be extremely long for such a high frequency service and would result in great passenger dissatisfaction. It is important to mention though that the calculations regarding passenger waiting times for the proposed service considered the published timetables of the preceding and following buses. Given that an acceleration threshold would be applied to all buses operating a route, the arrival and departure times of those two buses at stops along the route would also be altered.

We finally note that this work is not trying to solve the universal operation problem of a bus route that could arise from the application of an acceleration limit. Instead, it investigates the operational effects that might arise if the implementation of an acceleration limit of 1.0 $\mathrm{m} / \mathrm{s}^{2}$ were applied to a bus service in order to increase passenger safety and reduce non-collision injuries aboard buses. Looking at the journey time parameter in isolation, a round journey that could last up to 6 min longer when the safety-driven acceleration limit is imposed would not cause great dissatisfaction to passengers as currently some of them are waiting longer than expected (7.5 min). Moreover, not many passengers travel the entire length of a bus route, and in the case they do, they will experience an additional length of journey of around 3 min in each direction.

It is with no doubt that 6 min of additional journey time would have an impact on the operation of the bus service; one extra bus would be necessary to serve the same number of passengers of this bus route. However, the indirect benefits that will be enjoyed by both the bus passengers 
and operators (i.e. increased passenger safety, hence increased passenger satisfaction and thus patronage) have the potential to outweigh the cost of measures to meet reduced accelerations. Reducing bus accelerations would also have a great positive societal impact as the accessibility of bus services will increase and more people will become active members of a society that enjoys better health and well-being.

As part of a future work, real-time accelerations from the drive system of the preceding and following buses should be analysed in conjunction with the examined bus. This would draw a more complete picture of the impact that such an intervention would have on a bus service. Moreover, combining the proposed acceleration limit with bus priority measures would be more effective and would eliminate some of the limitations included in this work. Finally, calculating the cost of direct and indirect impacts resulting from such an intervention would provide evidence for a business case that aims to update current transport policies.

\section{Funding}

This research did not receive any specific grant from funding agencies in the public, commercial, or not-for-profit sectors.

\section{REFERENCES}

Adamski, A., Turnau, A., 1998. Simulation support tool for real-time dispatching control in public transport. Transportation Research Part A: Policy and Practice 32 (2), 73-87.

Berrebi, S. J., Watkins, K. E., Laval, J. A., 2015. A real-time bus dispatching policy to minimize passenger wait on a high frequency route. Transportation Research Part B: Methodological 81, 377-389.

Björnstig, U., Bylund, P., Albertsson, P., Falkmer, T., Björnstig, J., Petzäll, J., 2005. Injury events among bus and coach occupants: Non-crash injuries as important as crash injuries. IATSS research $29(1), 79-87$.

Browning, A., 1972. Human engineering studies of high speed pedestrian conveyors. Tech. rep., HM Stationery Office.

Cats, O., Larijani, A., Koutsopoulos, H., Burghout, W., 2011. Impacts of holding control strategies on transit performance: Bus simulation model analysis. Transportation Research Record: Journal of the Transportation Research Board (2216), 51-58.

CDC, 2019. Important facts about falls. www.cdc.gov/homeandrecreationalsafety/falls/adultfalls.html, (accessed 13-12-2019).

Ceder, A., Golany, B., Tal, O., 2001. Creating bus timetables with maximal synchronization. Transportation Research Part A: Policy and Practice 35 (10), 913-928.

Cevallos, F., Zhao, F., 2006. Minimizing transfer times in public transit network with genetic algorithm. Transportation Research Record: Journal of the Transportation Research Board (1971), 74-79.

Chen, Q., Adida, E., Lin, J., 2013. Implementation of an iterative headway-based bus holding strategy with real-time information. Public Transport 4 (3), 165-186.

Chen, X., Hellinga, B., Chang, C., Fu, L., 2015. Optimization of headways with stop-skipping control: a case study of bus rapid transit system. Journal of advanced transportation 49 (3), 385-401.

Daganzo, C. F., 2009. A headway-based approach to eliminate bus bunching: Systematic analysis and comparisons. Transportation Research Part B: Methodological 43 (10), 913-921.

Daganzo, C. F., Pilachowski, J., 2011. Reducing bunching with bus-to-bus cooperation. Transportation Research Part B: Methodological 45 (1), 267-277. 
Designed to move, 2015. Designed to move: Active Cities. http://e13c7a4144957cea5013f2f5ab26d5e83af3ea377013dd602911.r77.cf5.rackcdn.com/resources/pdf/en/active-cities-fullreport.pdf, (accessed 02-11-2018).

Dorn, M., 1998. Jerk, acceleration and the safety of passengers. Technology for Business Needs. Presented at the International Congress Railtech, Birmingham, 24th-26th November.

Eberlein, X. J., Wilson, N. H., Bernstein, D., 2001. The holding problem with real-time information available. Transportation science 35 (1), 1-18.

Eurostat, 2018. European statistics - transport. https://ec.europa.eu/eurostat/web/transport/data/database, (accessed 02-11-2018).

Frank, L. D., Andresen, M. A., Schmid, T. L., 2004. Obesity relationships with community design, physical activity, and time spent in cars. American journal of preventive medicine 27 (2), 87-96.

Fu, L., Liu, Q., Calamai, P., 2003. Real-time optimization model for dynamic scheduling of transit operations. Transportation Research Record: Journal of the Transportation Research Board (1857), $48-55$.

Fu, L., Yang, X., 2002. Design and implementation of bus-holding control strategies with real-time information. Transportation Research Record: Journal of the Transportation Research Board (1791), $6-12$.

Gkiotsalitis, K., 2020. Bus holding of electric buses with scheduled charging times. IEEE Transactions on Intelligent Transportation Systems.

Gkiotsalitis, K., Cats, O., 2018. Reliable frequency determination: Incorporating information on service uncertainty when setting dispatching headways. Transportation Research Part C: Emerging Technologies $88,187-207$.

Gkiotsalitis, K., Eikenbroek, O. A., Cats, O., 2019a. Robust network-wide bus scheduling with transfer synchronizations. IEEE transactions on intelligent transportation systems.

Gkiotsalitis, K., Maslekar, N., 2018a. Multiconstrained timetable optimization and performance evaluation in the presence of travel time noise. Journal of Transportation Engineering, Part A: Systems 144 (9), 04018058.

Gkiotsalitis, K., Maslekar, N., 2018b. Towards transfer synchronization of regularity-based bus operations with sequential hill-climbing. Public transport 10 (2), 335-361.

Gkiotsalitis, K., Wu, Z., Cats, O., 2019b. A cost-minimization model for bus fleet allocation featuring the tactical generation of short-turning and interlining options. Transportation Research Part C: Emerging Technologies 98, 14-36.

Green, J., Jones, A., Roberts, H., 2014. More than a to b: the role of free bus travel for the mobility and wellbeing of older citizens in london. Ageing \& Society 34 (3), 472-494.

He, S.-X., Dong, J., Liang, S.-D., Yuan, P.-C., 2019. An approach to improve the operational stability of a bus line by adjusting bus speeds on the dedicated bus lanes. Transportation Research Part C: Emerging Technologies 107, 54-69.

Ibarra-Rojas, O. J., Rios-Solis, Y. A., 2012. Synchronization of bus timetabling. Transportation Research Part B: Methodological 46 (5), 599-614.

Jansson, K., Pyddoke, R., 2010. Quality incentives and quality outcomes in procured public transportcase study stockholm. Research in Transportation Economics 29 (1), 11-18.

Karekla, X., 2016. Improving Accessibility of Public Transport Systems: the Influence of Double-Decker Bus Acceleration on Passenger Movement. Ph.D. thesis, University College London. 
Karekla, X., Tyler, N., 2018a. Maintaining balance on a moving bus: The importance of three-peak steps whilst climbing stairs. Transportation Research Part A: Policy and Practice 116, 339-349.

Karekla, X., Tyler, N., 2018b. Reducing non-collision injuries aboard buses: passenger balance whilst walking on the lower deck. Safety science 105, 128-133.

Karekla, X., Tyler, N., 2019. Reducing non-collision injuries aboard buses: passenger double support time whilst climbing the stairs. Safety Science 112, 152-161.

Kendrick, D., Drummond, A., Logan, P., Barnes, J., Worthington, E., 2015. Systematic review of the epidemiology of non-collision injuries occurring to older people during use of public buses in high income countries. Journal of Transport \& Health 2 (3), 394-405.

Koehler, L. A., Kraus, W., Camponogara, E., 2011. Iterative quadratic optimization for the bus holding control problem. IEEE Transactions on Intelligent Transportation Systems 12 (4), 1568-1575.

Li, J.-Q., Song, M. K., Li, M., Zhang, W.-B., 2009. Planning for bus rapid transit in single dedicated bus lane. Transportation research record 2111 (1), 76-82.

Liu, Z., Yan, Y., Qu, X., Zhang, Y., 2013. Bus stop-skipping scheme with random travel time. Transportation Research Part C: Emerging Technologies 35, 46-56.

London Travel Watch, 2010. Bus passengers' priorities for improvements in london. http://www.londontravelwatch.org.uk/documents, (accessed 02-11-2018).

Lu, H., Burge, P., Heywood, C., Sheldon, R., Lee, P., Barber, K., Phillips, A., 2018. The impact of real-time information on passengers value of bus waiting time. Transportation Research Procedia 31, $18-34$.

Newell, G. F., Potts, R. B., 1964. Maintaining a bus schedule. In: Australian Road Research Board (ARRB) Conference, 2nd, 1964, Melbourne. Vol. 2.

NHS, 2017. The incidence and costs of inpatient falls in hospitals. https://improvement.nhs.uk/resources/incidence-and-costs-inpatient-falls-hospitals/, （accessed $13-12-2019)$.

O'Neill, D., 2016. Towards an understanding of the full spectrum of travel-related injuries among older people. Journal of Transport \& Health 3 (1), 21-25.

Randall, E. R., Condry, B. J., Trompet, M., Campus, S. K., 2007. International bus system benchmarking: Performance measurement development, challenges, and lessons learned. In: Transportation Research Board 86th Annual Meeting, 21st-25th january.

Sale, A., 2007. Acceleration rate management test programme. Internal report No. MBK 07/0023. Tech. rep., Transport for London.

Shang, H.-Y., Huang, H.-J., Wu, W.-X., 2019. Bus timetabling considering passenger satisfaction: An empirical study in beijing. Computers \& Industrial Engineering 135, 1155-1166.

Shatnawi, N., Al-Omari, A. A., Al-Qudah, H., 2020. Optimization of bus stops locations using gis techniques and artificial intelligence. Procedia Manufacturing 44, 52-59.

Strathman, J. G., Wachana, P., Callas, S., 2010. Analysis of bus collision and non-collision incidents using transit its and other archived operations data. Journal of safety research 41 (2), 137-144.

Sun, A., Hickman, M., 2005. The real-time stop-skipping problem. Journal of Intelligent Transportation Systems 9 (2), 91-109.

Sun, S., Szeto, W., 2019. Optimal sectional fare and frequency settings for transit networks with elastic demand. Transportation Research Part B: Methodological 127, 147-177. 
TfL, 2017. Bus routes and borough reports. https://www.tfl.gov.uk/forms/14144.aspx, (accessed: 27-072017).

TfL, 2018. Transport for London budget 2018-19. http://content.tfl.gov.uk/transport-for-london-budget2018-19.pdf, (accessed 02-11-2018).

Trompet, M., Liu, X., Graham, D., 2011. Development of key performance indicator to compare regularity of service between urban bus operators. Transportation Research Record: Journal of the Transportation Research Board (2216), 33-41.

Verbas, İ. Ö., Frei, C., Mahmassani, H. S., Chan, R., 2015. Stretching resources: sensitivity of optimal bus frequency allocation to stop-level demand elasticities. Public Transport 7 (1), 1-20.

Wei, M., Sun, B., 2017. Bi-level programming model for multi-modal regional bus timetable and vehicle dispatch with stochastic travel time. Cluster Computing 20 (1), 401-411.

Welding, P., 1957. The instability of a close-interval service. Journal of the operational research society 8 (3), 133-142.

WHO, 2018a. More active people for a healthier world. http://www.who.int/ncds/prevention/physicalactivity/gappa, (accessed 02-11-2018).

WHO, 2018b. Physical activity. http://www.who.int/en/news-room/fact-sheets/detail/physical-activity, (accessed 02-11-2018).

Xuan, Y., Argote, J., Daganzo, C. F., 2011. Dynamic bus holding strategies for schedule reliability: Optimal linear control and performance analysis. Transportation Research Part B: Methodological 45 (10), 1831-1845.

Yan, S., Chi, C.-J., Tang, C.-H., 2006. Inter-city bus routing and timetable setting under stochastic demands. Transportation Research Part A: Policy and Practice 40 (7), 572-586.

Yu, B., Yang, Z., Yao, J., 2010. Genetic algorithm for bus frequency optimization. Journal of Transportation Engineering 136 (6), 576-583.

Yu, Y., Ye, Z., Wang, C., 2015. Study of bus stop skipping scheme based on modified cellular genetic algorithm. In: CICTP 2015. pp. 2397-2409.

Zeng, W., Miwa, T., Morikawa, T., 2020. Eco-routing problem considering fuel consumption and probabilistic travel time budget. Transportation Research Part D: Transport and Environment 78, 102219.

Zhang, H., Zhao, S., Cao, Y., Liu, H., Liang, S., 2017. Real-time integrated limited-stop and short-turning bus control with stochastic travel time. Journal of Advanced Transportation 2017.

Zhao, J., Dessouky, M., Bukkapatnam, S., 2006. Optimal slack time for schedule-based transit operations. Transportation Science 40 (4), 529-539. 